\title{
Resonance and morphological stability of tidal basins
}

\author{
Willem T. Bakker ${ }^{\text {a }}$, Huib J. de Vriend ${ }^{\mathbf{b}}$ \\ ${ }^{a}$ Netherlands Centre for Coastal Research (on secondment from Rijkswaterstaat, The Hague), Delft University of \\ Technology, Stevinweg 1, 2628 CN Delft, The Netherlands \\ ${ }^{b}$ University of Twente, School of Management Studies, Department of Civil Engineering and Management, P. O. Box 217, \\ 7500 AE Enschede, The Netherlands
}

Received 16 February 1994; revision accepted 10 November 1994

\begin{abstract}
The paper describes the concept of a network model for the morphological behaviour of a near-resonant multipleinlet tidal basin, as part of a model system which includes the barrier island coasts and the outer deltas. It addresses the question whether a small interference somewhere in such a basin can have major effects on sediment transport and morphology elsewhere in the system.

In order to explain the basic ideas of the model, only the main tidal constituent $\left(\mathrm{M}_{2}\right)$ and the associated topographyinduced residual current are considered, not the overtides. Furthermore, the model concerns only non-cohesive sediment (sand).

In spite of these simplifications, the model concept is shown to be effective, in that it results in a morphological evolution equation for each branch of the network and a picture of the influence of each branch on the resonancesensitivity of the system as a whole.
\end{abstract}

\section{Introduction}

A significant part of the world's coastline consists of systems of barrier islands and tidal inlets. The maintenance of such coasts is notoriously difficult, especially if the inlets are fixed by manmade works (e.g. jetties). Moreover, the basin usually contains a sensitive system of channels and intertidal areas of great ecological value, which is easily disturbed by anthropogenic effects. An accelerated rise of the mean sea level is another threat, because it is still unclear whether the intertidal flats will be able to follow the sea level. If so, the increased sediment demand of the basin may have detrimental effects on the barrier islands, especially if they have to be kept in place (e.g. because of inhabitation).

These are all good reasons to investigate the morphological behaviour of these systems, and to try and predict their response to human interference and exogenous changes. This may explain the long history of tidal inlet research, from purely empirical work (O'Brien, 1931, 1969) and classification (Hayes, 1979), via mathematical analyses (Escoffier, 1940, 1977; Van de Kreeke, 1990a,b) to highly sophisticated 3-D morphodynamic simulation models (Wang et al., 1994).

By definition, simulation models work at the time scale of the constituent physical processes, in this case the tide. Hence they are rather timeconsuming and difficult to control if they have to cover the time scales of decades or more which are typical for inlet systems. These models yield encouraging results on the channel dynamics, but they have not yet been able to reproduce the empirical equilibrium relationships for the cross- 
section of the gorge (Bruun and Gerritsen, 1960; Jarrett, 1976), the intertidal area (Renger, 1978; Renger and Partenscky, 1980) or the volume of the outer delta (Walton and Adams, 1976). This has created a demand for a more aggregated type of model, which works at the time and length scales of the relevant morphological evolutions and includes the equilibrium state.

One line of development was initiated in The Netherlands, stimulated by the need to have longterm predictions of the evolution of the Wadden Sea. It starts from the idea that a barrier island coast can be considered as a compound dynamic system of tidal basins, inlets, deltas and island coasts (Fig. 1). For each of these subsystems we know a number of empirical parameters of the equilibrium state (also see Eysink et al., 1992), and in some cases we can even estimate the time scale of evolution. Based on this information and physical process knowledge, and with the support of sophisticated simulation models, a set of semiempirical models was developed for a single-inlet system far from resonance, one for the basin (Van
Dongeren and De Vriend, 1994) and one for the outer delta combined with the adjacent island coasts (De Vriend et al., 1994).

As yet, these models have not been coupled, which means that they have to be forced artificially at the interface, so in the gorge. Moreover, they apply to non-resonant single-inlet systems only. The latter is not sufficient for the Western Wadden Sea, which is a near-resonant multiple-inlet system. Therefore, a special model concept for this type of system is developed, at the same time trying to avoid the interfacing problem in the gorge.

The present paper outlines the concept of this model, which is based on Lorentz's (1926) successful linear network model of the tide in the Western Wadden Sea. Without claiming that all quantitatively important processes are included, the basic principle of the morphodynamic evolution model is explained. The concept is shown to have the potential to become a practically useful tool, which can easily be extended with other transport mechanisms and which can be integrated with an outer delta and coastline model.

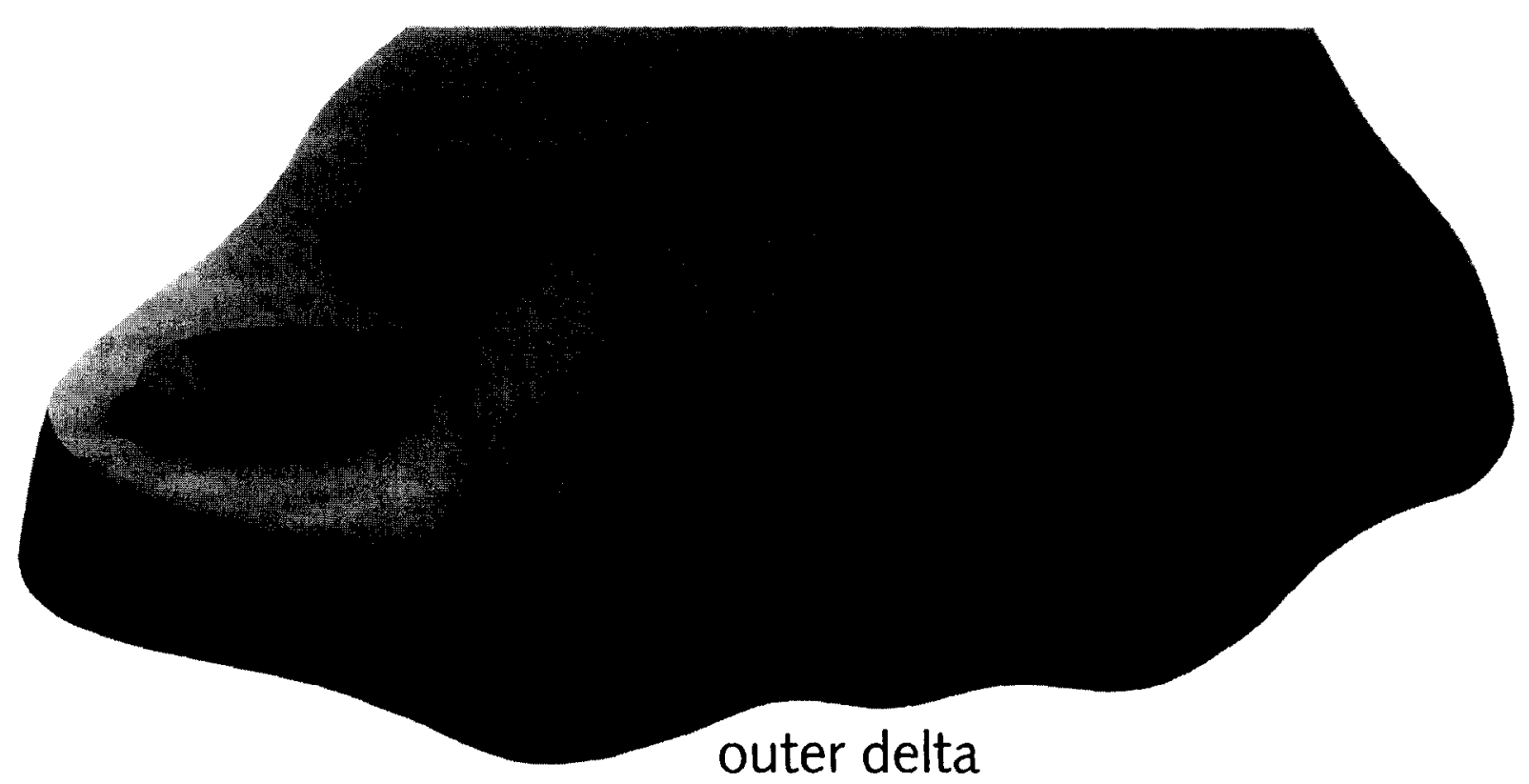

Fig. 1. A barrier island coast can be considered as a compound dynamic system of tidal basins, inlets delta's and island coasts. The final system model in mind will comprehend all the elements, shown in Fig. 1. 


\section{Approach}

The geometrical schematization of the basin area is a channel network (Fig. 2), which divides the model domain into a number of elements (sections). Each channel cross-section consists of one or more deep and one or more shallow parts. The former represents the water and sediment conveying channels in the relevant section, the latter the intertidal flats, which are all assumed to lie at mean water level. Transfer of water and sediment between sections is concentrated in the nodal points, so there is no direct transfer between shoal areas. By letting some network branches stick through the inlets into the sea, the undisturbed tide can be imposed at the seaward ends of these branches. Thus the interfacing problem in the gorge can be avoided.

The tide in this network is described with a numerical version of Lorentz's (1926) linear tidal model (Jallah and Bakker, 1994). This linearity is instrumental to the concept, so nonlinearity effects have to be included via perturbation methods, effective stress models or otherwise. In order to keep complexity within bounds, the inclusion of these elements is postponed till a later stage, irrespective of their quantitative importance. Only the topographical rectification effect of flooding and drying is taken into account.

The sediment transport rate is described by a simple power-law formula, which relates the transport rate in an element to the local flow velocity.

The key element of the model is the morphological evolution equation per section, which is based on the sediment balance and elaborated using the hydrodynamic and sediment transport equations. The bed level in the channels is the only remaining dependent variable. This is also the only morpho-

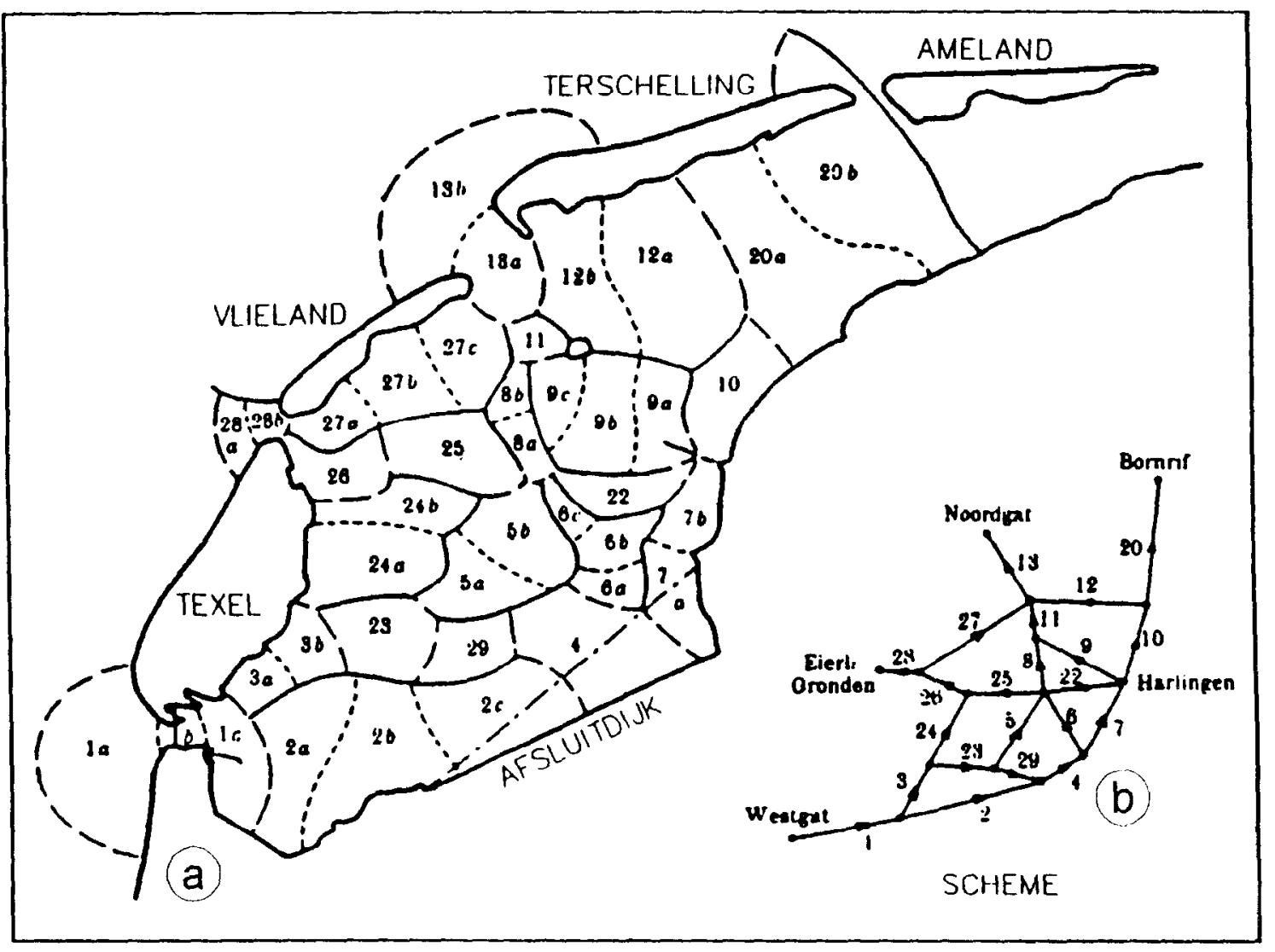

Fig. 2. Schematization of the Western Wadden according to Lorentz et al. (1926). (a) Topography; (b) schematization. 
logical variable in the model, since vertical accretion or erosion of the shoals is precluded by fixing them all at the mean water level. Horizontal extension of the shoals is precluded as long as the channel and shoal widths are fixed.

The model consists of the morphological evolution equations for all channels in the network. They form a coupled mathematical system, in which the coefficients are derived from a tidal model run on the initial bed topography. In the next sections, the various parts of this evolution model will be discussed in further detail.

\section{Model for a single channel section}

The equivalent of the present model for a single channel section has been described in depth by Bakker (1995). In order to have a reference for the network model, a brief summary will be given here.

The constituting equations of the Lorentz (1926) model for a single channel are the linearized momentum equation averaged over the channel cross-section:

$\frac{\partial Q}{\partial t}+g A \frac{\partial \zeta}{\partial x}+f Q=0$

and the corresponding equation of continuity:

$B_{\mathrm{s}} \frac{\partial \zeta}{\partial t}+\frac{\partial Q}{\partial x}=0$

in which $x=$ spatial co-ordinate along the channel,

$t=$ time,

$Q=$ flow rate,

$g=$ acceleration due to gravity,

$A=$ cross-sectional area below mean water level,

$\zeta=$ water surface elevation,

$f=$ friction factor (linearized friction model), and

$B_{s}=$ storage width.

These equations are linear in $\zeta$ and $Q$, so they can be solved analytically for a harmonic tidal wave in a channel of finite length. If complex exponentials are used (e.g. Ippen, 1966, ch. 10.4), the result is a complex amplitude, $z$ and a complex flow rate, $Q_{\mathrm{c}}$ as function of $x$ and $t$. The modulus of these complex parameters indicates the amplitude, the argument the phase lag of the tidal wave.
A typical solution reads:

$\zeta(x, t)=\operatorname{Re}\left\{z(x) e^{i \omega t}\right\}$

for the water level and a corresponding expression for the flow rate.

The transport in the channel is assumed to be described by the third-power transport formula. If we consider a combination of a tide and a weak steady current, the tidally averaged transport rate per unit channel width follows from:

$\langle S\rangle=\frac{3}{2} a \delta \hat{u}^{3}+O\left(\delta^{3}\right)$

in which $\langle S\rangle=$ the tidally averaged transport rate $\left[L^{2} T^{-1}\right]$,

$a=$ constant of proportionality in the transport formula,

$\delta=$ ratio between the mean current in the part of the cross-section below low water and the tidal velocity amplitude,

$\hat{u}=$ amplitude of the tidal current velocity.

When combined with the sediment balance equation:

$(1-\varepsilon) \frac{\partial h}{\partial t}-\frac{\partial\langle S\rangle}{\partial x}=0$

in which $\varepsilon$ is the porosity of the bed, this equation can be elaborated to a tide-averaged evolution equation. For small perturbations to a given morphology this can be written as:

$\frac{\partial \Delta h}{\partial t}=f n c t(\Delta h)$

in which $\Delta h$ is a perturbation to the water depth below mean water level in the deep part of the channel. The most important assumptions underlying this equation are

- the bottom of the deep part of the channel is the only morphologically active part of crosssection, i.e. shoals remain of the same size and at the same level,

- the time scale of the morphological evolution is large compared with the tidal period.

Eq. 6 describes the stability of the channel morphology against perturbations. Depending on whether the right-hand part is decreasing or 
increasing as $\Delta h$ increases, the system is morphologically stable or unstable.

\section{Network model: tidal module}

Via the harmonic solution of the hydrodynamic equations for a single channel, the tide at the one end of each section is linked to that at the other end. In a network model, we have to close the mathematical system via another set of relationships, between the channels which meet at each nodal point. Per nodal point, these relationships can be found from the continuity requirement (the sum of all flow rates toward a node equals zero) and the compatibility requirement (the water levels at the ends of all branches in one node are the same).

Thus we find a system of $N$ equations with $N$ unknown complex amplitudes $z$, which can formally be written as:

$$
[A] z=Z
$$

in which [A] is the $N \times N$ system matrix, $z$ is the vector of unknown amplitudes and $Z$ is the source vector, which contains the seaward boundary conditions. The solution in a nodal point $n$ can be written as:

$z_{\mathrm{n}}=\sum_{k=1}^{K} b_{k n} Z_{k}$

in which $K$ is the number of seaward boundary points (everywhere else $Z=0$ ). The coefficients $b_{k n}$ are elements of the inverse system matrix $[\mathbf{A}]^{-1}$.

Eq. 8 shows that the tide in any nodal point is a linear combination of the tides at the seaward boundaries. For a multiple-inlet system this means that the contribution to the tidal amplitude can mapped for each of the inlets separately. As an example, Fig. 3 shows these influence functions for each of the inlets of the Western Wadden Sea, as computed with the network model shown in Fig. 2.

\section{Topography-induced rectification}

Tidal asymmetry is an important transport agent in the channel--shoal system inside the basin. The topography-induced generation of overtides (hypsometry effect; Boon and Byrne, 1981; Speer and Aubrey, 1985) is one important effect, which probably plays a role in the equilibrium state as a balancing agent against the sediment importing shallow-water asymmetry at the seaward end of the model (Van Dongeren and De Vriend, 1994). Another topography-induced effect is rectification, due to which there is a residual circulation between the shoals and the channels.

For the time being, overtides will be left out of consideration (see Discussion). The rectification effect is taken into account by treating shoals and channels separately. As the shoals are assumed to lie at mean water level, they fall dry during part of the tide. The net flux of water (mass flux) over a shoal of width $B_{\text {sh }}$ during the other part of the tide is given by:

$\left\langle Q_{\mathrm{sh}}\right\rangle=\frac{1}{4} B_{\mathrm{sh}} \hat{z} \hat{u}_{\mathrm{sh}} \cos \phi_{\mathrm{sh}}$

in which $\hat{u}_{\text {sh }}$ denotes the amplitude of the tidal velocity on the shoal, and $\phi_{s h}$ the phase difference between the vertical and the horizontal tide. Note that if this phase difference is $90^{\circ}$, as in the case of a deep-water tide, the net flux goes to zero.

In the channels, the residual mass flux above LW, due to the phase relationship between water level and velocity, follows from:

$$
\left\langle Q_{\mathrm{ch}}+\right\rangle=\frac{1}{2} B_{\mathrm{ch}} \hat{z} \hat{u}_{\mathrm{ch}} \cos \phi_{\mathrm{ch}}
$$

in which the suffix $\mathrm{ch}^{+}$refers to the tide-induced mass flux in the channel ${ }^{1}$. In order to satisfy the overall mass balance, there has to be another

\footnotetext{
${ }^{1}$ Lorentz (1926) and Thijsse (1955?) implement composite profiles of tidal channels in their tidal computation. These are first schematized as a set of parallel channels and then replaced by an hydraulically equivalent rectangular channel. The equivalent channel is characterized by its width, depth and friction factor $f$. The width equals the sum of the widths of its constituent channels. The depth and friction factor are determined by its discharge, equal to the sum of the discharges in the constituent channels (taking phase differences into account). The same vertical tide in all parallel channels is assumed. Thus, amplitude and phase of the horizontal tide in each of the constituent channels result from the Lorentz computation. These values are used in Eqs. 9 and 10
} 

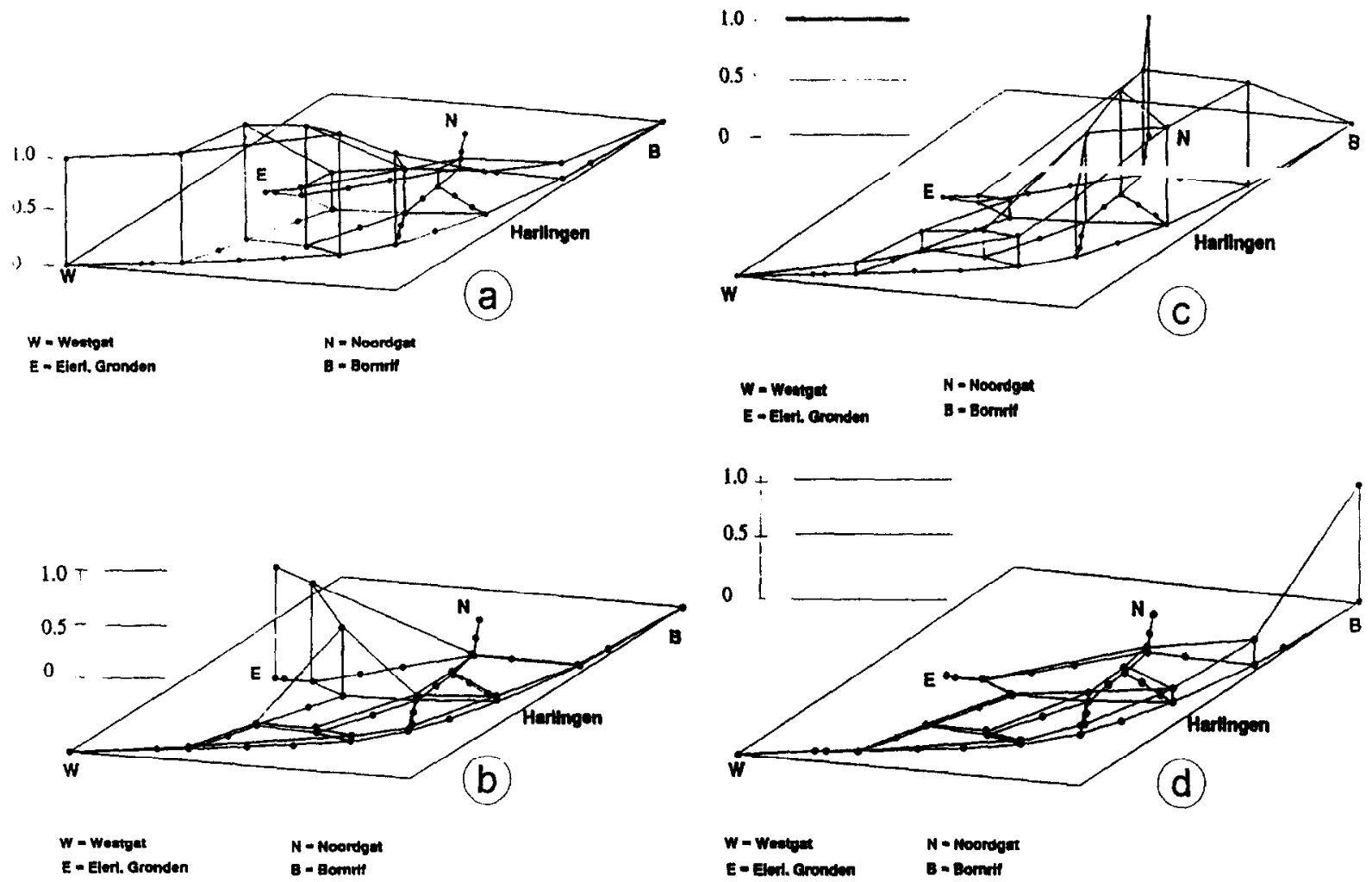

Fig. 3. Influence coefficients for the various inlets of the Western Wadden Shoals. (a) The inlct Westgat; (b) the inlet Eierlandse Gronden; (c) the inlet Noordgat; (d) the inlet Bornrif.

residual current, which is assumed to be concentrated entirely in the channels. From this point of view the channels function as "drains" of the basin. In the same vein, the spatial mass flux gradients, e.g. due to a gradient in the phase difference $\phi_{\mathrm{ch}}$, can be considered as producing "rain" which flows off via the "drains" (Fig. 4).

Fig. 5 gives an impression of the distribution of the mass-flux induced "rain" and the corresponding run-off flow through the network. The former follows directly from the mass flux gradients, the latter is the result of a steady-flow computation with a nonlinear numerical model with the "rain" as a source term. The above tidal model was used to compute the mass flux and the tidal velocities needed to determine the enhanced bottom friction in the steady flow model.

In the particular case of the Western Wadden Sea, with its high shoals and almost perfect watersheds, one can say for most points beforehand to

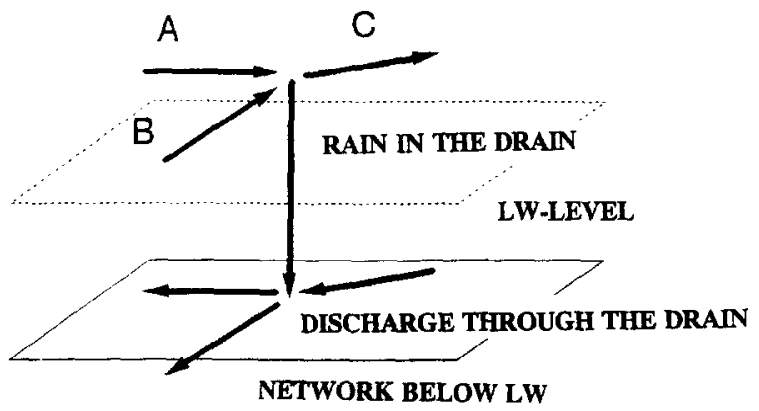

Fig. 4. The tide-averaged flux of water above the LW-line should be transferred into a "rain in the drain" and subsequently discharged through the parts of the channels below LW.

which inlet the rain will flow. Hence the run-off pattern is primarily a matter of continuity. In other situations, however, other forcing mechanisms may determine the run-off pattern.

The mass flux is only one of the tidal rectification 

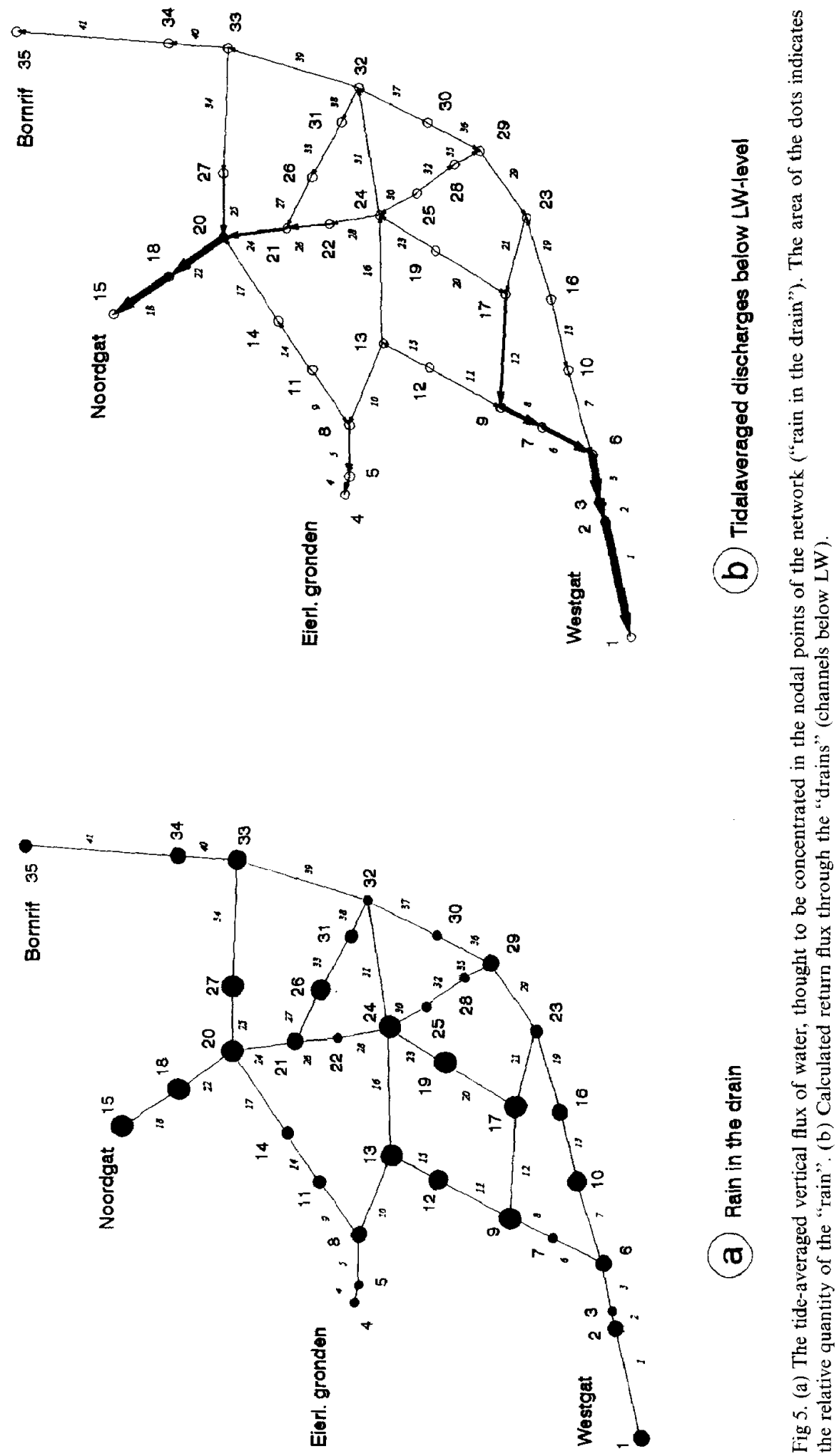
mechanisms. The advection terms in the momentum equations can also give rise to a residual current, via what is usually called the tidal stress (Nihoul and Ronday, 1975). Once we use a separate model to compute the residual current, this tidal stress can easily be included, just as a wind stress or any other external forcing.

The idea of separating the oscillatory and the mean part of the current is common practice in wave-driven current modelling. There the mean flow is driven by exactly the same mechanisms: mass flux, effective stress and bed stress enhancement (for instance, see Fredsoe and Deigaard, 1992). Nonlinear tidal simulation models usually describe the depth-averaged velocity as a function of time and then integrate over the tide to compute the residual current. Thus the mass flux, tidal stress and bed shear stress effects are automatically included, at least if the model is provided with a proper flooding and drying procedure. The practice of depth-averaged flow modelling, however, has also led to the widely spread picture that the residual tidal current should have the same vertical distribution as the instantancous velocity (usually logarithmic). This is not necessarily the case, due to the phase coupling between water level and velocity.

The residual current model has been verified (Dijkstra, 1994) against a fully nonlinear tidal model $^{2}$ of the mouth of the Western Scheldt, a tidal estuary in the south of The Netherlands, also with a pronounced topography. The geometry is much simpler than in the Wadden Sea case, viz. a single channel which bifurcates in the mouth of the estuary. For this geometry, the "rain and drain" approach gives a good approximation of the residual velocities according to a fully nonlinear model (see Fig. 6)

\section{Morphodynamic evolution equations}

For explanatory reasons, the procedure which leads to the set of morphodynamic evolution equa-

\footnotetext{
${ }^{2}$ Used is the DUFLOW model (cf. Spaans et al., 1989), designed for unsteady flow computations in networks of open channel courses. Non-linear friction and inertia terms are implemented. The program allows for composite profiles. The deepest part of the channels should be below LW.
}

tions will only be written up in formal terms. The physical considerations and the mathematical operations per section are essentially the same as in the previous case of a single section. For further details: see Bakker (1995).

Following the same procedure as for the singlesection case, the residual transport gradient per section can formally be written as

$\left.\frac{\partial\langle S\rangle}{\partial x}\right|_{j}=F_{j}\left(z_{j}^{+}, z_{j}^{-}, \delta_{j}, \hat{u}_{j}\right)$

in which $F_{j}$ denotes a functional relationship which follows straightforwardly from the expression for the residual transport (cf. Eq. 4). The superfixes \pm and - refer to either end point of the sections.

This expression can be substituted into the sediment balance equation for section $j$, to yield:

$\left.\frac{\partial h}{\partial t}\right|_{j}=\left.\frac{1}{1-\varepsilon} \frac{\partial\langle S\rangle}{\partial x}\right|_{j}=\frac{1}{1-\varepsilon} F_{j}\left(z_{j}^{+}, z_{j}^{-}, \delta_{j}, \hat{u}_{j}\right)$

The quantities $z^{+}, z, \delta$ and $\hat{u}$ depend in principle on the water depth in each of the $M$ channel sections of the model. So if we consider small perturbations to a given morphological state, we can write $F_{j}$ in the perturbed state as:

$F_{j} \approx F_{j}^{(0)}+\sum_{\ell=1}^{M} \Delta h_{\ell}\left(\frac{\partial F_{j}}{\partial h_{\ell}}\right)_{0}$

in which the superfix " $(0)$ " refers to the given state. Hence, the system of morphological evolution equations for all sections can be written as:

$\frac{\partial \boldsymbol{\Delta} \boldsymbol{h}}{\partial t}=\frac{1}{1-\varepsilon}[\boldsymbol{B}] \Delta \boldsymbol{h}$

in which $\mathbf{h}$ denotes the vector of water depths and $[B]$ is a matrix operator. The quantity $\Delta h$ is defined as $h-h^{(0)}$. So if we know the matrix, this equation indicates whether a perturbation to the given state in a section tends to grow or decay, and how it interacts with changes in the other sections. Hence it can be used to investigate the stability and the inherent dynamic behaviour of the morphological system.

The elements of the matrix can be determined 
by formally writing them as:

$\frac{\partial F_{j}}{\partial h_{\ell}}=\frac{\partial F_{j}}{\partial z_{j}^{+}} \frac{\partial z_{j}^{+}}{\partial h_{\ell}}+\frac{\partial F_{j}}{\partial z_{j}^{-}} \frac{\partial z_{j}^{-}}{\partial h_{\ell}}+\frac{\partial F_{j}}{\partial \delta_{j}} \frac{\partial \delta_{j}}{\partial h_{\ell}}+\frac{\partial F_{j}}{\partial \hat{u}_{j}} \frac{\partial \hat{u}_{j}}{\partial h_{\ell}}$

in which the variation of $z^{+}, z^{-}, \delta$ and $\hat{u}$ with $h_{\ell}$ has to be determined from multiple runs of the tidal model. In view of Eq. 8, this means that we have to determine the coefficients $\beta_{k n \ell}$ in:

$\frac{\partial z_{n}}{\partial h_{\ell}}=\sum_{\mathrm{k}=1}^{K} \frac{\partial b_{k n}}{\partial h_{\ell}} Z_{k}=\sum_{k=1}^{K} \beta_{k n \ell} Z_{k}$

and similar coefficients for the relative residual current velocity, $\delta$, and the tidal velocity amplitude $\hat{u}$. Since we know $b_{k n}$ and can derive the tidal velocity in a section from the complex tidal amplitude at either end, this requires $M$ runs of both the tidal and the residual current model.

Thus $M$ equations have been obtained, which describe the morpho-dynamic behaviour of the system in a linear approximation. These equations can be utilized to investigate various aspects of this behaviour, such as:

- the stability of each channel to perturbations in its own depth, without interaction with the other channels; the sign of the corresponding element on the main diagonal of the matrix [B] determines whether a channel is stable in this respect;

- the influence of depth perturbations in each of the channels on the resonance properties of each inlet;

- the character of mutual interaction of groups of channels, or of all channels in the system;

- the stability of the system as a whole.

Of course, the equations can also be used to simulate the system's morphodynamic behaviour on computer.

In the following, the model is utilized to assess the sensitivity of the resonance properties of the Western Wadden Sea to a small amount of change in the depth of each channel section. The marker quantity is the flow rate through each inlet channel $k(k=1, . ., 4)$, which can be written as:

$Q_{k} \sim Z_{k}\left(1-b_{k k}\right) \frac{1+e^{4 i \theta}}{2 i}$

The first factor herein represents the tide at the seaward boundary, the second the feedback from the basin, and the third the effect of friction in the gorge: the angle $\theta$ is zcro for an infinite flat bottom and $\pi / 4$ when the bottom roughness is infinitely large. So the interesting factor from a resonance point of view is the second one, in which $b_{k k}$ denotes the coefficient in the relationship (8) between the tide in the nodal point just inside the inlet and the tide at sea. The modulus of $b_{k k}$ goes to infinity if the system is resonant.

In order to assess which channel has the strongest influence on the resonance properties of inlet $k$, the quantity:

$\frac{\partial}{\partial h_{\ell}}\left|1-b_{k k}\right|, \quad l=1, \ldots, M$

is determined for each channel $l$. Fig. 7 shows the results for each of the inlets. Apparently, the pattern of influences is not quite trivial. Some channels are much more resonance-prone than others, and deepening of some even tends to reduce the resonance factor. The pictures for the Westgat and the Noordgat inlets are largely complementary, in that interferences which reduce the resonance factor for the Westgat tend to increase the one for the Noordgat and conversely. Also, the Westgat turns out to be much more resonanceprone than the other inlets.

The next step would be to assess the stability of the "critical" channel, taking its effect on the resonance factor of the relevant inlet into account. In that case, the rate of change of the transport gradient in the channel can be written as a function of $\Delta h_{\ell}$ and $1-b_{k k}$ only:

$\Delta \frac{\partial S}{\partial x}=f\left(\Delta h_{\ell}, 1-b_{k k}\right)$

Hence the morphodynamic equation for this channel reads:

$\Delta\left(\frac{\mid h_{\ell}}{\partial t}\right)=\alpha \Delta h_{\ell}+\beta \frac{\partial\left|1-b_{k k}\right|}{\partial h_{\ell}} \Delta h_{\ell}=\gamma \Delta h_{\ell}$

in which $\alpha$ and $\beta$ are proportionality constants and in which $\gamma$ originates from addition.

The sign of the factor $\gamma$ determines whether this interaction is stable: if $\gamma$ is positive it is unstable 

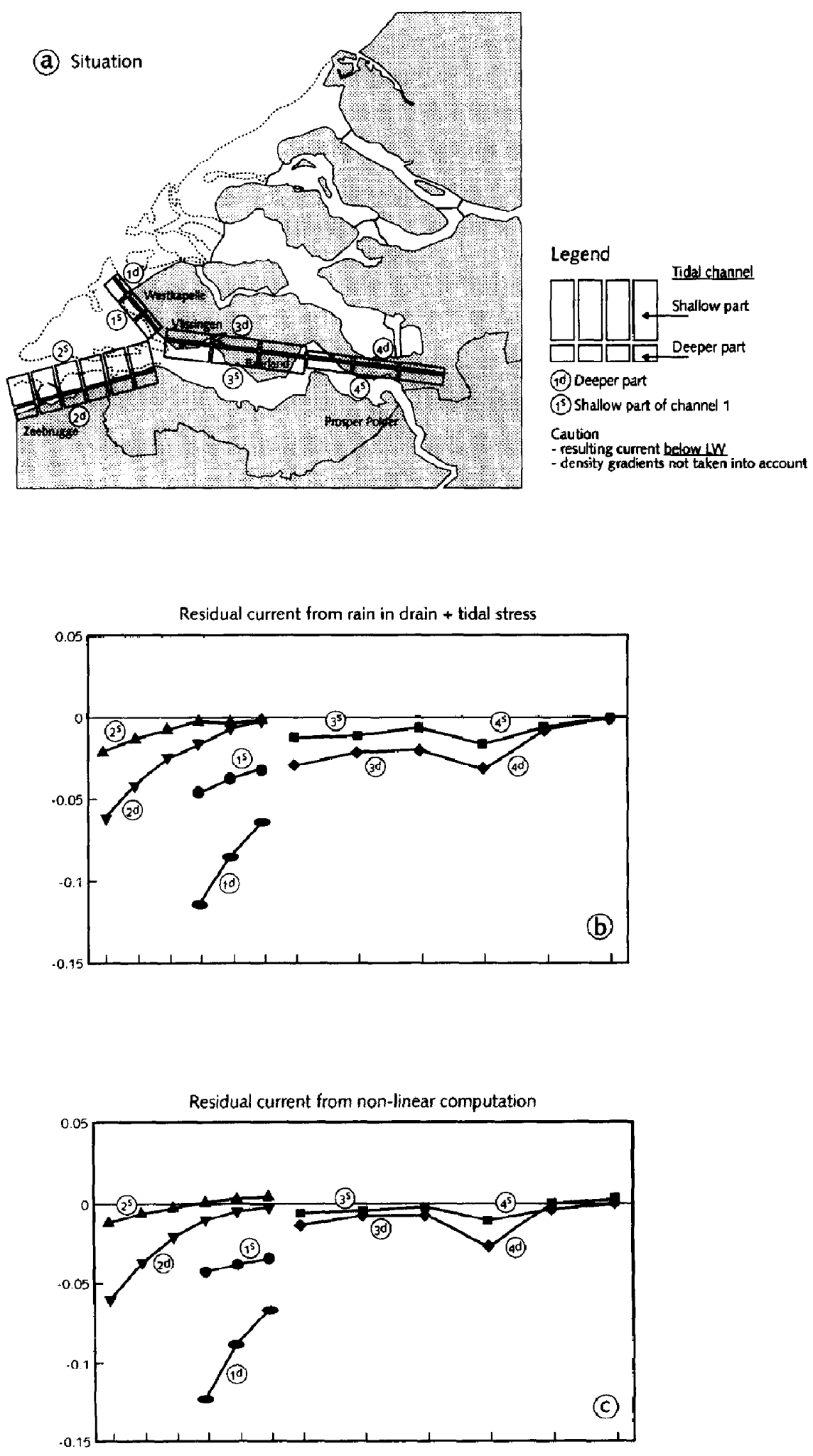
and a small change in the channel depth can lead to increasing resonance of the basin. The quantifcation for the western Wadden sea remains to be made.

\section{Discussion}

The model concept which is described in the foregoing should be considered as a first step on the way to long-term morphodynamic modelling of near-resonant multiple-inlet tidal basins. It shows the essence of the procedure to be followed in order to derive a set of morphodynamic evolution equations which encompasses the hydrodynamics without going through a time-consuming simulation process.

The route which was taken herein led to the analysis of the effect of morphological changes on the resonance properties of the basin, and to a linear stability analysis of the morphodynamic system. Another possibility which this concept offers is to consider perturbations to the equilibrium state and describe the evolution from a given initial state to that equilibrium state.

Clearly, the model is still far from complete. The lack of tidal stresses in the residual current model is one gap which has to be filled, but a possibly even more important one concerns the effects of overtides, especially those generated by the flooding and drying of the intertidal flats (Speer and Aubrey, 1985; Van Dongeren and De Vriend, 1994). Yet the residual current should not be disregarded as a transport agent. Van de Kreeke and Robaczewska (1993) show for the Ems estuary, further eastward in the Wadden Sea, that the contribution of the residual current to the transport even exceeds that due to the M4 overtide. Besides, it must be not too difficult to include the overtides in the model, once it is possible to parameterize their topographical generation. Their inclusion will probably change the outcome of the model, but not the essence of the approach.

The morphological flexibility of the model should probably be extended. Especially if the model is to be utilized for long-term predictions, the intertidal zone has to be made morphologically active, in the vertical (flats' level) as well as in the horizontal (flats' area vs. channel area). This probably implies that the tidal model runs have to be repeated from time to time, according to the rate of change of the shoals and the channel planform. The frequency of these runs will be much smaller, however, than in the case of straightforward simulation (cf. De Vriend et al., 1993).

It is also clear that the model needs further validation. The strong parallels of the "rain and drain" concept with the well-established practice in wave-driven current modelling gives some confidence, and so does the comparison with the nonlinear numerical model for the Western Scheldt, but this is not enough. If the model has to run for the Western Wadden Sea, it also has to be validated for that area. A promising option is a comparison with the residual current maps produced by Ridderinkhof (1988a,b). However, in all comparisons the Eulerian kind of definition of residual current in the present paper should be kept in mind.

An interesting extension of the model might be to include fine (cohesive?) sediment. Here the proportion between the transporting agents is quite different, because the sediment is distributed much more evenly over the vertical. Transport of material above the LW-level becomes crucial as well. The near-bed residual current is therefore less important; thus the rain-in-the-drain concept looses its physical meaning. The tidal asymmetry plays a key role (cf. Dronkers, 1986). Another approach, like the one of Krol (1990) might be more appropriate. Ways of combining both methods is a challenge for the future.

Fig. 6. Tidal motion in the Western Scheldt (a) has been simulated in a Y-shaped model. The cross-section of each of the channels consists of a deep and a shallow part. Calculated results of tide-averaged currents $(\mathrm{in} \mathrm{m} / \mathrm{s})$ in the various parts of the channel are displayed in (b and c); in (b) the rain-in-the-drain method, in (c) the result of a non-linear network model (DUFLOW) was averaged over the tide. In both computations density gradients were not (yet) taken into account: the check is mathematical, not physical. 

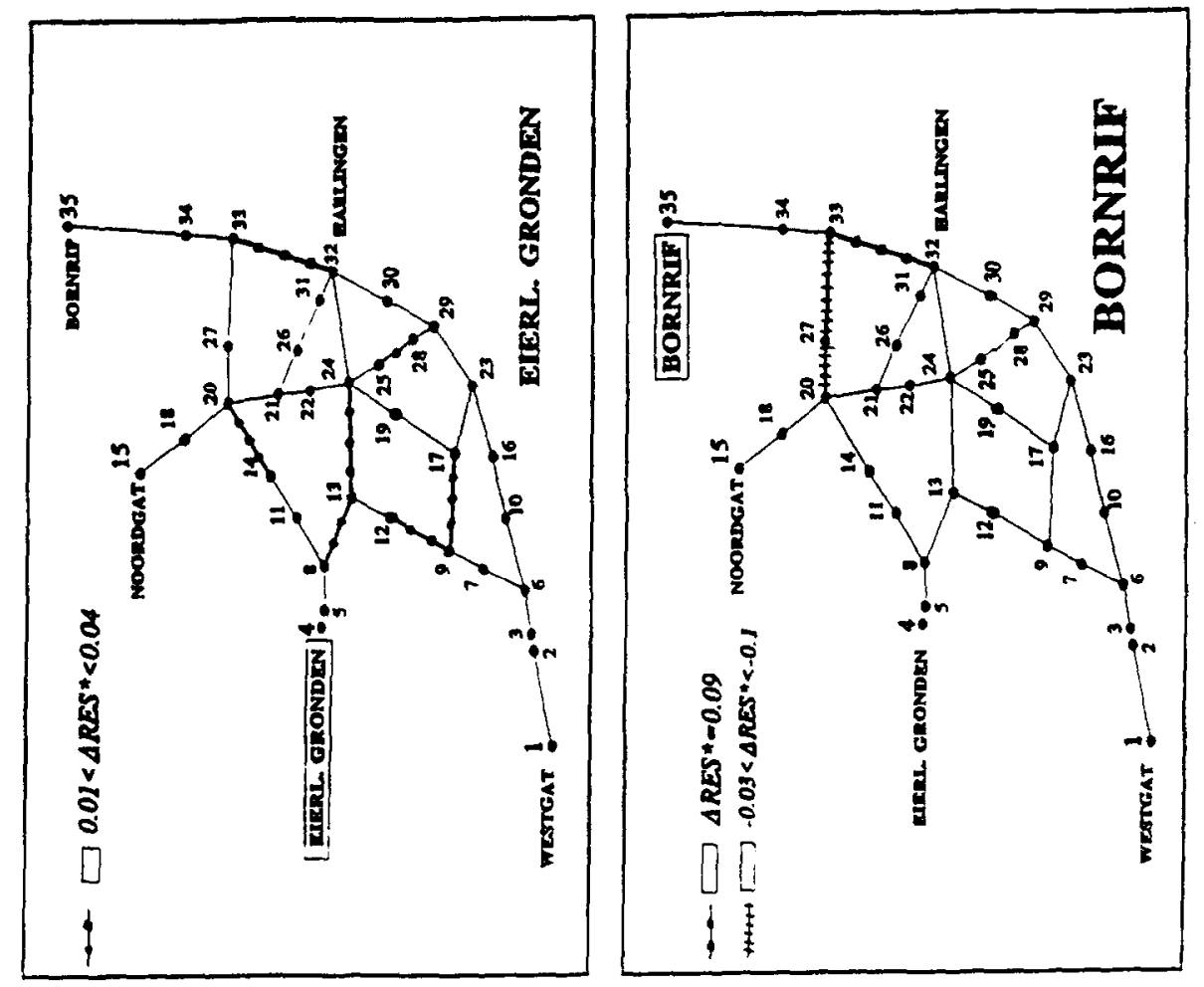

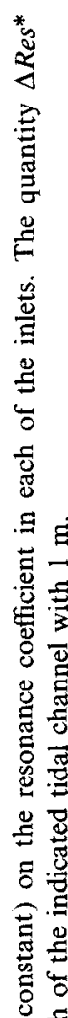
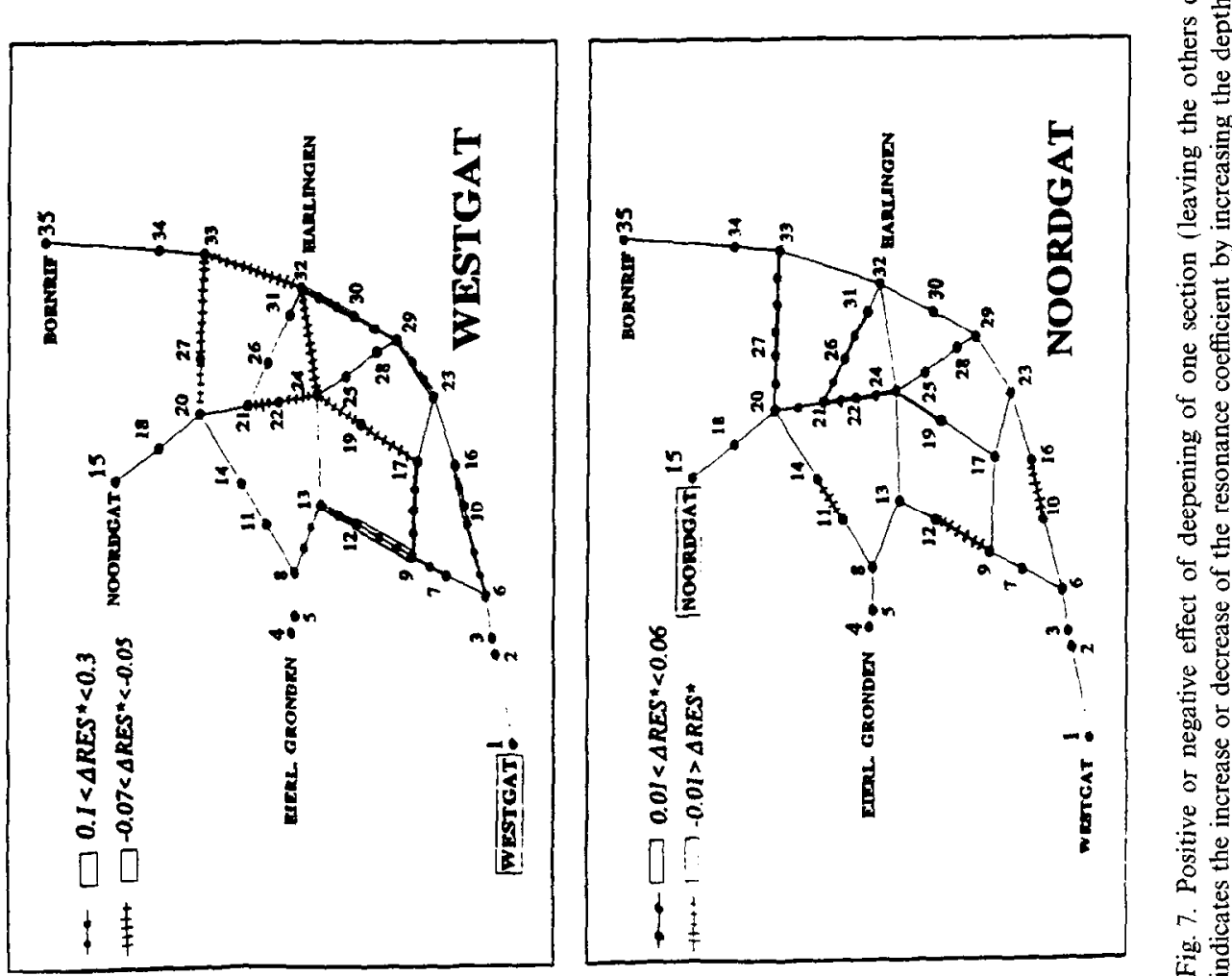


\section{Conclusions}

A model concept is described which has the potential to be the basis of a long-term morphological model for near-resonant multiple-inlet tidal basins. The key element is a set of morphological evolution equations, one for each channel section of the network. These equations are based upon a simplified tidal model in which the oscillatory tidal motion is separated from the residual current. Thus the topography-induced rectification via the residual mass flux above low water can be taken into account via the "rain and drain" approach.

The model was shown to give an indication of the resonance-sensitivity of each of the inlets of the Western Wadden Sea, The Netherlands, to a change of depth in each of the channels in the basin. It was also shown to provide the possibility to assess the morphodynamic stability of the channel system, and to extend it to a long-term prediction model for this type of basins. Via its connection with the open sea, it provides the possibility to be integrated with a model which describes the morphology of the outer delta and the barrier island coasts.

\section{Acknowledgements}

The authors acknowledge the importance of the references Jallah and Bakker (1994) and Bult (1994) and Dijkstra (1994), which are underlying reports of the present paper. The contributions of those authors, and their consent to reproduce Figs. 3 and 7 (A.N. Jallah M.Sc.), Fig. 5 (G. Bult) and Figs. 4 and 6 (A.G.H. Dijkstra) are gratefully acknowledged.

\section{References}

Bakker, W.T., 1995. Effect of tidal resonance on the morphology of Wadden and estuaries. Submitted.

Boon, J.D. and Byrne, R.J., 1981. On basin hypsometry and the morphodynamic response of coastal inlet systems. Mar. Geol., 40: 27-48.

Bruun, P. and Gerritsen, F., 1960. Stability of Coastal Inlets. North Holland, Amsterdam, 123 pp.

Bult, G., 1994. Wadden and Estuaries: Science and
Management. Rijkswaterstaat, RIKZ, Res. Strategy Dep. and CAT Enschede, Inter. Rep. (In Dutch.)

De Vriend, H.J., Capobianco, M., Chesher, T., De Swart, H.E., Latteux, B. and Stive, M.J.F., 1993. Approaches to long-term modelling of coastal morphology: a review. Coastal Eng., 21 (1/3): 225-269.

De Vriend, H.J., Bakker, W.T. and Bilse, D.P., 1994. A morphological behaviour model for the outer delta of mixedenergy tidal inlets. Cuastal Eng., 23(3/4): 305-327.

Dijkstra, A.G.H., 1994. Modeling tides and residual currents in the Western Scheldt. Rijkswaterstaat, RIKZ, and CAT Enschede, Inter. Rep. (In Dutch.)

Dronkers, J., 1986. Tidal asymmetry and estuarine morphology. Neth. J. Sea Res., 20: 117-131.

Escoffier, F.F., 1940. The stability of tidal inlets. Shore Beach, $8(4): 114-115$.

Escoffier, F.F., 1977. Hydraulics and stability of inlets. U.S. Army Corps Eng., CERC G.I.T.I. Rep. 13, Vicksburg, MI, 72 pp.

Eysink, W.D., Biegel, E.J. and Hoozemans, F.J.M., 1992. Impact of sea level rise on the morphology of the Wadden Sea in the scope of its ecological function; investigations on empirical morphological relations. Delft Hydraulics, ISOS*2 Rep., H 1300.

Fredsoe, J. and Deigaard, R., 1992. Mechanics of Coastal Sediment Transport. (Advanced Series in Ocean Eng., 3.) World Scientific, Singapore, 369 pp.

Hayes, M.O., 1979. Barrier island morphology as a function of tidal and wave regime. In: S.P. Leatherman (Editor), Barrier Islands from the Gulf of St. Lawrence to the Gulf of Mexico. Academic Press, New York, pp. 1-27.

Ippen, A.T., 1966. Estuary and Coastline Hydrodynamics. McGraw-Hill, New York.

Jallah, A.N. and Bakker, W.T. 1994. Lorentz on PC. Delft Univ. Technol., Dep. Hydraul. Eng., Inter. Rep.

Jarrett, J.Г., 1976. Tidal prism-inlet area relationships. U.S. Army Corps Eng., CERC G.I.T.I. Rep. 3, Vicksburg, MI, 55 pp.

Krol, M., 1990. The method of averaging in partial differential equations. Ph.D. Thesis, Univ. Utrecht.

Lorentz, H.A., 1926. Report State Commission Zuiderzee 1918-1926. Algemeene Staats-drukkerij, The Hague. (In Dutch.)

Nihoul, J.C.J. and Ronday, F.C., 1975. The influence of the tidal stress on the residual circulation. Tellus, 27: 484-489.

O'Brien, M.P., 1931. Estuary tidal prisms related to entrance areas. Trans. ASCE, 1(8): 738-739.

O'Brien, M.P., 1969. Equilibrium flow areas of inlets on sandy coasts. Proc. ASCE, J. Waterw. Harbors Coastal Eng., 15(WW1): 43-52.

Renger, E., 1978. Two-dimensional stability analysis of tidal basins and tidal flats of larger extent. In: Proc. 16th Int. Conf. Coastal Eng., ASCE, New York, pp. 1971-1983.

Renger, E. and Partenscky, H.W., 1980. Sedimentation processes in tidal channels and tidal basins caused by artificial constructions. In: Proc. 17th Int. Conf. Coastal Eng., ASCE, New York, pp. 2481-2494. 
Spaans, W., Booij, N., Praagman, N., Noorman, R. and Lander, J., 1989. DUFLOW; a micro-computer package for the stimulation of one-dimensional unsteady flow in open channel systems,

Speer, P.E. and Aubrey, D.G., 1985. A study of non-linear tidal propagation in shallow inlet/estuarine systems, part II: theory. Est. Coastal Shelf Sci., 21: 207-224.

Thijsse, J.Th., 1955(?). Theory of Tides. Int. Course in Hydraulic Engineering. (Delft.)

Ridderinkhof, H., 1988a. Tidal and residual flows in the western Dutch Wadden I: numerical model results. Neth. J. Sea Res., 22: 1-22.

Ridderinkhof, H., 1988b. Tidal and residual flows in the western Dutch Wadden II: an analytical model to study a constant flow between connected tidal basins. Neth. J. Sea Res., 22: 185-198.

Van de Kreeke, J., 1990a. Can multiple inlets be stable? Estuarine Coastal Shelf Sci., 30: 261-273.

Van de Kreeke, J., 1990b. Stability analysis of a two-inlet bay system. Coastal Eng., 14: 481-497.

Van de Kreeke, J. and Robaczewska, K., 1993. Tide-induced transport of coarse sediment; application to the Ems estuary. Neth. J. Sea Res., 31(3): 209-220.

Van Dongeren, A.R. and De Vriend, H.J., 1994. A model of morphological behaviour of tidal basins. Coastal Eng., 22(3/4): 287-310.

Wang, Z.B., Louters, T. and De Vriend, H.J., 1995. Morphodynamic modelling for a tidal inlet in the Wadden Sea. Mar. Geol., 126: 000-000. (This volume.) 